\title{
Aplicación de acciones metodológicas innovadoras en Geografía: mirando en rural y urbano mediante las "células de trabajo"
}

\author{
CAROLINA DEL VALLE RAMOS \\ Universidad de Sevilla \\ Facultad de Geografía e Historia \\ Departamento de Geografía Humana \\ cdvalle@us.es \\ ORCID: https://orcid.org/0000-0001-5327-0790 \\ D.O.I.: http://dx.doi.org/10.12795/JDU.2018.i01.10 \\ Pp.: $177-197$
}

\section{Resumen}

La siguiente comunicación presenta los resultados obtenidos tras la realización de un Ciclo largo de Mejora Docente (CMDC) llevado a cabo en la asignatura de "Geografía Rural y Urbana", dentro del programa REFID (Red para la Formación y la Innovación Docente) que oferta la Universidad de Sevilla. El diseño metodológico aplicado se ha basado en lo que he denominado "células de trabajo", utilizando como marco teórico la teoría de los sistemas (Ludwig von Bertalanffy, 1950). Los resultados obtenidos, evaluados tanto al final de cada módulo como de la asignatura, ponen de manifiesto que la aplicación de estas acciones innovadoras favorecen no sólo la adquisición de contenidos sino 
también la satisfacción y percepción del alumnado con respecto a la docencia universitaria.

Palabras claves: Geografía Rural y urbana, Grado de Geografía y Gestión del Territorio, Docencia Universitaria, Experimentación Docente Universitaria, células de trabajo.

\section{Breve descripción del contexto de la intervención}

La experiencia innovadora que se presenta se realiza en la asignatura "Geografía Rural y Urbana", obligatoria de 2 - curso del Grado de Geografía y Gestión del Territorio, por lo que el ciclo abarca 30 horas totales. Esta asignatura se distribuye en 45 horas de teoría y 15 de prácticas, lo que supone un valor de 6 créditos ECTS. El ciclo de mejora abarcó las dos tipologías de clases. Hay que destacar que esta asignatura ya fue objeto de mejoras docentes durante el Curso académico 2016/2017, dentro de la realización del curso de iniciación del Programa de Formación e Innovación docente del profesorado, cuyos resultados se presentaron en las IV Jornadas de Formación e Innovación Docente realizadas en Sevilla en Diciembre del 2017. Con respecto a las características del alumnado de dicha asignatura, en este curso 2017/2018 estaban matriculados un total de 42 alumnos, de los que el 85\% aproximadamente han venido a clase con regularidad. De ellos, sólo había una alumna extranjera, procedente del Reino Unido, la cual ha mostrado un alto grado de integración con el resto de sus compañeros en todo momento.

Uno de los aspectos positivos de aplicar el CMD en esta asignatura, es el hecho de que se trata del mismo alumnado al que le he impartido en el cuatrimestre anterior otra asignatura también de carácter obligatorio. Ese contacto ya adquirido anteriormente ha facilitado en gran medida el trabajo, puesto que se ha partido de unos vínculos de conexión que producen un acercamiento entre el docente y el alumnado: los conocía y llamaba por sus nombres, hay un conocimiento de los grupos con más interés, los que menos, quién suele trabajar y quién no. En definitiva, acelera el clima de trabajo en el aula. 


\section{Diseño del Ciclo de Mejora}

\section{Mapa de contenidos y problemas o preguntas claves}

Aunque el mapa de contenidos que aparece realizado en la figura 1 abarca toda la asignatura, el ciclo de mejora (CDM) este curso académico ha sido aplicado únicamente a los últimos 5 bloques (elementos caracterizadores, representaciones cartográficas, paisajes, problemas y políticas y gestión). En cada uno de ellos se han trabajado de forma conjunta contenidos conceptuales, procedimentales y actitudinales, siguiendo como modelo metodológico un diseño propio del mismo que será analizado en el siguiente epígrafe. La forma utilizada para enlazar los tres tipos de contenidos citados anteriormente ha sido mediante la realización de preguntas claves. Los contenidos se estructuraron no solo en los bloques de trabajo, sino que se dividió el mismo en dos partes: una el ámbito urbano y, la otra, el ámbito rural. Se ha tratado por tanto de trabajar sobre la base de una serie de preguntas/problemas que enfrentaban dos escenarios algunas veces contrapuestos y otras veces complementarios. El territorio urbano y el rural por definición parecen ser opuestos, sin embargo, encontramos en ellos procesos o comportamientos que siguen las mismas pautas de desarrollo. Así pues, esta singularidad que aporta el territorio al contenido de la asignatura favorece la posibilidad de realizar un diseño de contenidos ampliamente relacionables entre sí. Las preguntas planteadas para cada bloque aparecen en la tabla 1.

Jornadas de Formación e Innovación Docente del Profesorado I № 1 (2018) Esta obra se distribuye con la licencia Creative Commons 

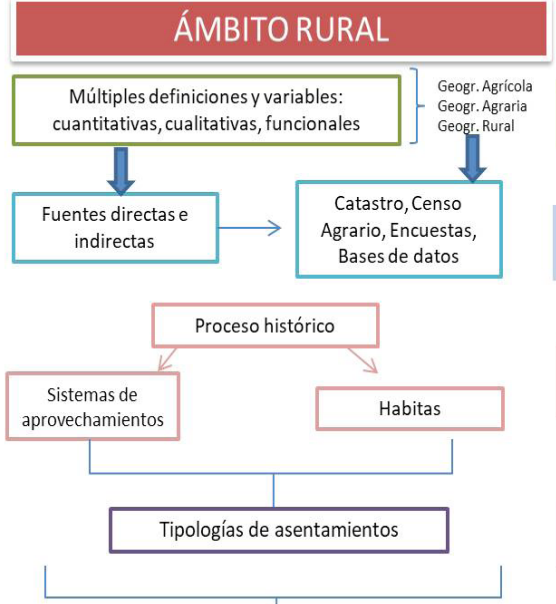

Paisajes agrarios mundiales y en España

Sociales y demográficos: envejecimiento despoblación...

Funcionales: aislamiento, urbanización,...

Medombientales: residuos, agricultura agresiva,

insostenibilidad mediambiental...

Documentos de planeamiento y gestión territorial
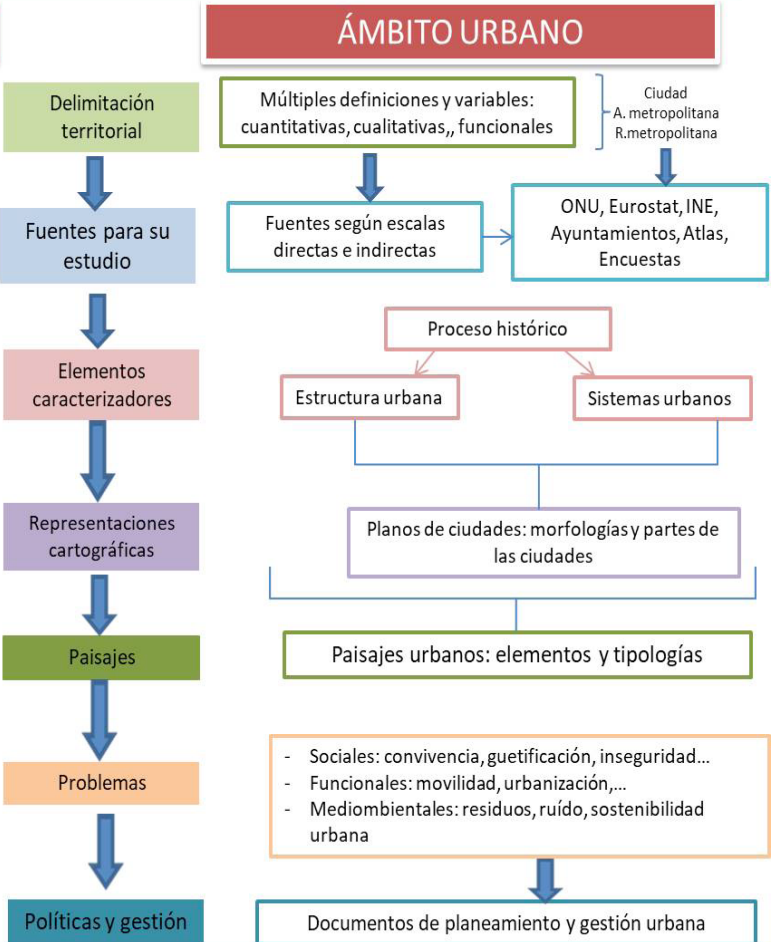

\section{CONTENIDOS CONCEPTUALES, PROCEDIMENTALES Y ACTITUDINALES}

Figura 1: Mapa de contenidos de la asignatura completa

\section{Tabla 1}

\section{Preguntas planteadas al inicio de cada bloque.}

\begin{tabular}{|l|l|}
\hline $\begin{array}{l}\text { Bloque 1: ¿Cuál es el límite que di- } \\
\text { ferencia el espacio rural del espa- } \\
\text { cio urbano? }\end{array}$ & $\begin{array}{l}\text { Bloque 2: Me ha contratado en } \\
\text { una Consultora para hacer un es- } \\
\text { tudio sobre el tipo de parcela- } \\
\text { rio de un ámbito (rural y urbano), } \\
\text { ¿de qué fuentes puedo obtener la } \\
\text { información? }\end{array}$ \\
\hline $\begin{array}{l}\text { Bloque 3: ¿Es igual la estructura ur- } \\
\text { bana que encontramos en un pue- } \\
\text { blo de 10.000 habitantes que en una } \\
\text { gran ciudad? ¿Por qué?. }\end{array}$ & $\begin{array}{l}\text { Bloque 4: ¿Qué escala utilizarías } \\
\text { para representar en un informe te- } \\
\text { rritorial un asentamiento rural y/o } \\
\text { urbano? }\end{array}$ \\
\hline
\end{tabular}

Jornadas de Formación e Innovación Docente del Profesorado I № 1 (2018) (C) (i) $\odot$ Esta obra se distribuye con la licencia Creative Commons Internacional (CC BY-NC-ND 4.0.) 
Bloque 5: ¿Qué elementos podemos analizar en dos fotografías, una rural y otra urbana, que sean singulares del paisaje característico de cada ámbito?
Bloque 6: ¿Qué tipo de problemas (sociales, funcionales, ambientales,) podemos encontrar en los espacios rurales y urbanos?

Bloque 7: Ante esos problemas, ¿hay algún sistema de planificación y/o gestión que los trate? ¿Cuáles y en qué forma?

\section{Modelo metodológico y secuencia de actividades}

Mis principales incertidumbres e inquietudes, y es por ello por lo que me decidí a realizar este proyecto, estaban generadas por el hecho de no conocer hasta qué punto la actividad docente llevada a cabo se estaba realizando de una manera óptima en lo que al proceso de enseñanza-aprendizaje se refiere. Incluso en algunos casos, los análisis realizados a los resultados de las encuestas voluntarias de calidad de la enseñanza que se realizan por parte de la Universidad de Sevilla, habían puesto de manifiesto en años anteriores algunas debilidades en el proceso de enseñanza, el cual había sido manifestado por el alumnado. Por otra parte, también había que destacar la existencia de una necesidad de renovación y conocimiento de nuevas metodologías docentes, así como de nuevas pautas que permitiesen "innovar" en el proceso de aprendizaje. El curso pasado se llevaron a cabo ciertas actividades que, mediante la utilización de nuevas herramientas metodológicas, formaron parte de ciclos de mejora de corta duración. Ante ello se comprueba la importancia de cambiar el diseño metodológico no sólo de una parte de la asignatura sino de la misma al completo. Mi metodología hasta ese momento seguía prácticamente unas pautas tradicionales en el proceso de enseñanza aprendizaje, basado fundamentalmente en la utilización de la clase magistral, la realización de algunas actividades prácticas por parte del alumnado y, finalmente, la realización de un examen 
para comprobar el nivel de adquisición de los contenidos teóricos. Ese era el modelo real. Seguía una secuenciación totalmente lineal y tradicional, sin salirme de ningún límite que me alterara mi "zona de confort" y que yo pudiese controlar sin problemas.

Durante este curso, he conseguido avanzar en el tipo de modelo metodológico aplicado. Como modelo ideal, plantearía el utilizado por Ralph Lynn y que Ken Bain explica en su libro "Lo que hacen los mejores profesores universitarios". Sin embargo, al reflexionar sobre el modelo metodológico que de forma real podía llevas a cabo, me encontré con el hecho de que, ya a priori, las dificultades estructurales eran realmente importantes. Es difícil encontrar una universidad española que proporcione al docente los medios y herramientas necesarios para llevar a cabo una educación y formación de su alumnado de forma excelente. Faltas de espacios adecuados para realizar ciertas actividades, deficiencias de infraestructuras y acondicionamientos para la utilización de nuevas tecnologías, ayudas a la docencia en lo que capital humano se refiere. Y a todo ello hay que unir la tipología y características del alumnado que nos solemos encontrar, donde suele haber a priori falta de motivación, de interés, etc.

En este caso, mi espacio asignado para las sesiones de clase ha sido el aula XX de la Facultad de Geografía e Historia. Un espacio estrecho y alargado, con bancas y mesas fijadas al suelo, que dificultaban ya a priori algunas de las actividades ha había diseñado para realizar. Contaba con proyector, acceso a internet y wifi, lo cual favorecía el poder acceder a información instantánea por parte del alumnado.

Con todo ello, el planteamiento de mi diseño metodológico para este curso se ha basado en lo que he denominado como "células de trabajo", que se encuentran interrelacionadas entre sí e imbuidas de dinamismo, siguiendo como marco teórico lo que sería el planteamiento 
de un sistema (teoría de los sistemas del biólogo austríaco Ludwig von Bertalanffy, 1950).

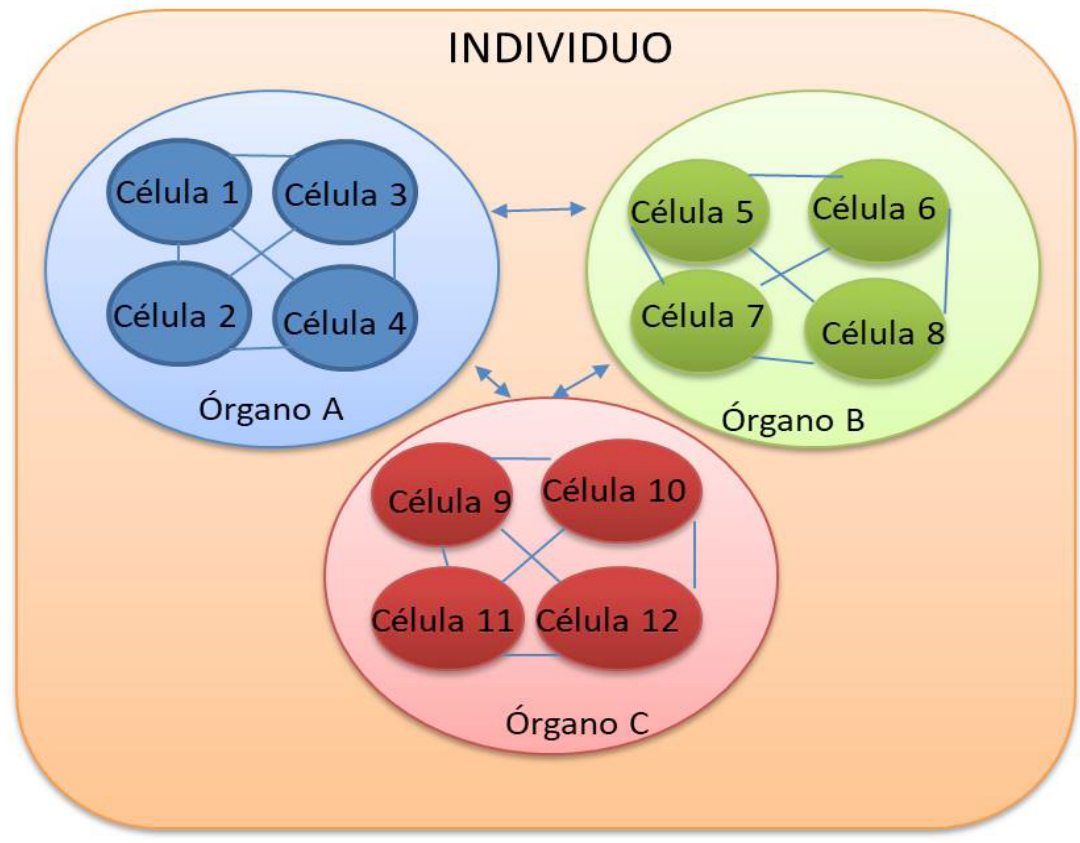

Figura 2: Diseño de la estructura metodológica de trabajo del alumnado en el desarrollo del CDM

Cada célula funciona de forma individual y autónoma, pero cuyo desarrollo-trabajo va a depender de otras células y, a su vez, va a influir en las demás que conforman el sistema. El planteamiento que les expliqué al alumnado fue el siguiente: "Un organismo está compuesto por miles de células cada una con su función. Cuando varias de éstas células se unen pueden conformar un órgano del individuo. Los distintos órganos hacen que el individuo viva sano y alcance se meta. Cada uno de vosotros vais a ser una célula de trabajo. Por tanto tenéis que realizar un trabajo autónomo y propio. Trabajareis algunas actividades por grupos formando "un órgano". De esa manera tendremos todos los órganos necesarios poder seguir viviendo sano y conseguir avanzar. Si una célula no funciona, afectará al órgano al que pertenezca, pero realmente estará 
afectando a todo el individuo. De esta manera el individuo puede morir (fracasar en la superación de los contenidos de la asignatura)".

Además, los contenidos (conceptuales, procedimentales y actitudinales) también son diseñados como un sistema. De esa forma, se favorece que el alumnado adquiera una visión global del funcionamiento, en este caso, del Territorio.

El tratamiento de cada uno de los bloques que se han trabajado (del bloque 3 al 7) estaba dividido de la siguiente manera:

\section{Módulo A_ Marcos conceptuales}

Los marcos conceptuales se comienzan a trabajar a partir de una encuesta previa pasada a todos los alumnos sobre conceptos e ideas claves del bloque. A partir de los resultados de esas encuestas se diseñan los contenidos conceptuales que son necesarios que el alumnado adquiera y la metodología a seguir, que en este caso ha estado dividida entre lecturas de bibliografía específica, clase teórica magistral y puesta en común de ideas y conceptos claves. Se corresponde con trabajo autónomo de la "célula".

\section{Módulo B_Actividades individuales}

Con las actividades individuales se ha pretendido que el alumnado adquiriese y desarrollara conocimientos procedimentales de análisis de procesos. El objetivo en este caso era que sean ellos mismos avanzaran en su escalera de conocimientos de forma autónoma. Se corresponde con trabajo autónomo de la "célula".

\section{Módulo C_ Actividades grupales}

Durante todo el ciclo de mejora se han destinado una serie de horas al trabajo grupal. De esta manera se fomenta 
no sólo la adquisición o desarrollo del conocimiento conceptual, sino también procedimental y en gran medida el actitudinal. Para ello, se ha utilizado el diálogo/debate/ puesta en común de ideas como herramienta fundamental. Además, se ha realizado un trabajo grupal con proyección a la divulgación pública del conocimiento y la cultura (que es explicado más adelante). Se corresponde con trabajo en grupo de forma colaborativa (unión de varias células que conforman órganos permitiendo que el organismo haya avanzado en la consecución de un objetivo).

\section{Módulo D_ Trabajo fuera de aula}

El trabajo fuera de aula ha permitido tanto trabajar sobre el terreno conceptos y procedimientos de la realidad del territorio, como asentar las bases para reflexionar sobre marcos conceptuales. Podemos diferenciar entre la salida de campo y las sesiones realizadas en la Facultad pero fuera del aula. En éste caso la célula debía funcionar alternando trabajo autónomo con unión en órganos.

\section{Módulo E_Autoevaluación}

Se han diseñado unas pautas de trabajo de autoevaluación por parte de cada alumno apoyándonos en el uso de las nuevas tecnologías, exactamente de aplicaciones llevadas a cabo con terminales móviles. La autoevaluación se ha realizado tanto de forma individual, lo que permite que cada alumno sea consciente después de cada bloque de su evolución en la adquisición de contenidos; pero también de forma global, lo que ha permitido una reflexión sobre el funcionamiento de cada órgano dentro del sistema. De esta manera la secuencia del modelo metodológico llevado a cabo puede verse en la tabla 2. 


\section{Tabla 2}

\section{Secuencia seguida en el desarrollo del modelo}

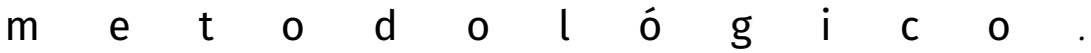

1. Planteamiento del problema: Se planteará al alumnado la pregunta/ problema a la que deben dar respuesta.

2. Presentación de posibles herramientas metodológicas a seguir para dar respuesta al problema.

3. Trabajo autónomo de cada célula: desarrollo del contenido procedimental por parte del alumno.

4. Trabajo del "órgano". Grupal y colaborativo

5.Puesta en común de las posibles respuestas al problema: el "individúo" avanza (o se estanca)

6. Desarrollo de argumentaciones a la resolución del problema. Estará centrado principalmente en el desarrollo y adquisición de los contenidos conceptuales y actitudinales. Este apartado será básicamente desarrollado por mí.

7. Autoevaluación: Al final de cada pregunta planteada y resuelta se realizará una autoevaluación individual, grupal y comentarios finales que dejen abierta la puerta a la siguiente pregunta a plantear para la siguiente sesión.

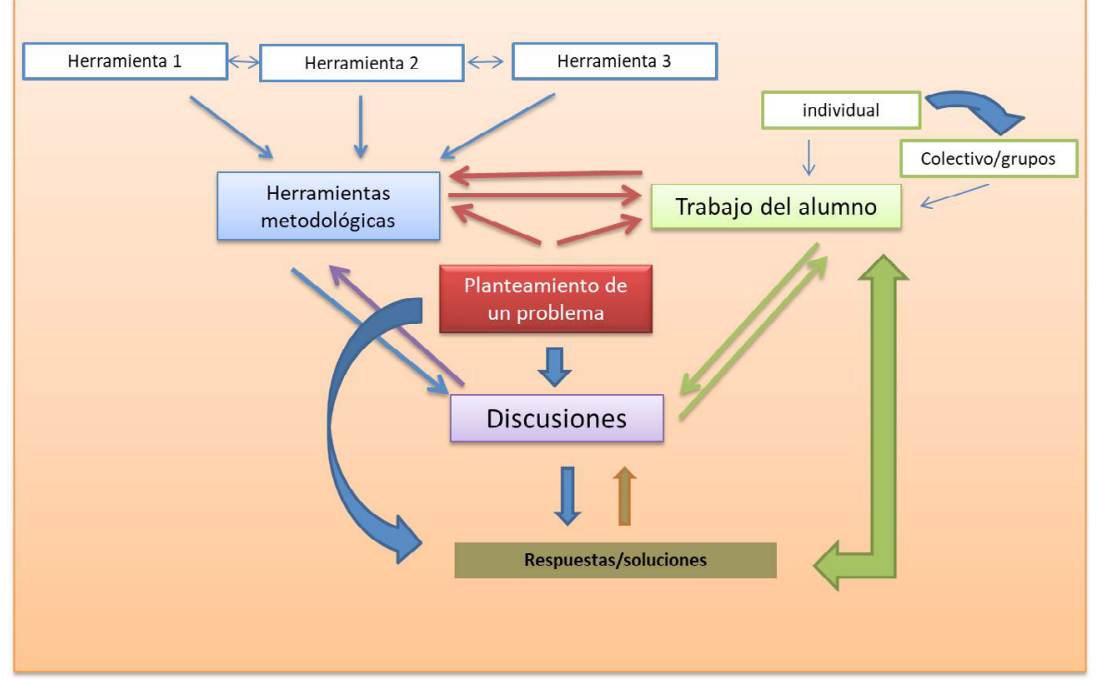

Figura 3: Modelo metodológico aplicado en cada bloque

Siguiendo ese modelo se han realizado la tabla 1 que muestra cómo se han ido realizando secuencialmente el 
conjunto de actividades durante toda la duración del Ciclo de Mejora Docente:

\title{
Tabla 3
}

\author{
Cronograma de actividades desarrolladas en el CMD \\ de la asignatura "Geografía Rural y Urbana"
}

\begin{tabular}{|c|c|}
\hline SEMANA Y BLOQUE & ACTIVIDADES \\
\hline $\begin{array}{l}\text { Semana } 12 . \\
\text { Bloque 3: ¿Es igual la es- } \\
\text { tructura urbana que en- } \\
\text { contramos en un pueblo de } \\
10.000 \text { habitantes que en } \\
\text { una gran ciudad? ¿Por qué?. }\end{array}$ & $\begin{array}{l}\text { *Cuestionario previo sobre contenidos del bloque. } \\
\text { *Visionado de documental: "Soy de pueblo, y qué } \\
\text { ?. Debate y reflexión. } \\
\text { *Trabajo individual con fuentes estadísticas ofi- } \\
\text { ciales del IECA, INE y EUROSTAT. } \\
\text { *Trabajo en grupo y presentaciones orales sobre } \\
\text { la realidad en distintos escenarios europeos. }\end{array}$ \\
\hline $\begin{array}{l}\text { Semana } 13 . \\
\text { Bloque 3: ¿Es igual la es- } \\
\text { tructura urbana que en- } \\
\text { contramos en un pueblo de } \\
10.000 \text { habitantes que en } \\
\text { una gran ciudad? ¿Por qué?. }\end{array}$ & $\begin{array}{l}\text { * Taller conceptual: realización individual de es- } \\
\text { quemas de contenidos (conceptuales y procedi- } \\
\text { mentales). Puesta en común, trabajo en grupos y } \\
\text { debate. } \\
\text { * Realización de material cartográfico que mues- } \\
\text { tra resultados del taller conceptual realizado en la } \\
\text { sesión anterior. } \\
\text { *Autoevaluación individual y en grupo: mediante } \\
\text { Kahoot se autoevalúan ellos mismos y también } \\
\text { de forma grupal } \\
\text { *Cuestionario final contenidos del bloque, para } \\
\text { evaluar la escalera de aprendizaje. }\end{array}$ \\
\hline $\begin{array}{l}\text { Semana } \mathbf{1 4 .} \\
\text { Bloque 4: ¿Qué escala uti- } \\
\text { lizarías para representar } \\
\text { en un informe territorial } \\
\text { un asentamiento rural y/o } \\
\text { urbano? }\end{array}$ & $\begin{array}{l}\text { *Cuestionario previo sobre contenidos del bloque. } \\
\text { *Visionado de documental: "Escalas territoriales y } \\
\text { administrativas". Debate y reflexión. } \\
\text { *Trabajo individual con noticias periodísticas: } \\
\text { Analizamos la escala utilizada en distintos perió- } \\
\text { dicos para representar territorios de sus noticias: } \\
\text { ámbitos internacionales, nacionales, regionales y } \\
\text { locales. } \\
\text { *Trabajo en grupo y presentaciones orales so- } \\
\text { bre la escala que se utilizan en distintos pla- } \\
\text { nes territoriales y urbanísticos para representar } \\
\text { Territorios. }\end{array}$ \\
\hline
\end{tabular}

Jornadas de Formación e Innovación Docente del Profesorado | № 1 (2018) Esta obra se distribuye con la licencia Creative Commons Reconocimiento-NoComercial-SinObraDerivada Internacional (CC BY-NC-ND 4.0.) 


\section{Semana 15.}

Bloque 4: ¿Qué escala utilizarías para representar en un informe territorial un asentamiento rural $\mathrm{y} / \mathrm{o}$ urbano?

\section{Semana 16.}

Bloque 5: ¿Qué elementos podemos analizar en dos fotografías, una rural y otra res del paisaje característico de cada ámbito? urbana, que sean singula-

* Taller conceptual: realización individual de esquemas de contenidos (conceptuales y procedimentales). Puesta en común, trabajo en grupos y debate.

* Realización de inventario sobre la cartografía que debería tener un informe territorial (se toman distintas escalas) apoyándonos en el resultados del taller conceptual realizado en la sesión anterior.

*Autoevaluación individual y en grupo: mediante Kahoot se autoevalúan ellos mismos y también de forma grupal

*Cuestionario final contenidos del bloque, para evaluar la escalera de aprendizaje.

*Cuestionario previo sobre contenidos del bloque. *Visionado de fragmento de la película "Memorias de África" para reflexionar sobre el concepto de Paisaje.

*Trabajo individual: realización de una fotografía de un espacio rural y analizar los elementos que aparecen en la misma.

*Trabajo en grupo y presentaciones orales de análisis de paisajes rurales: factores y elementos.

\section{Semana 17.}

Bloque 5: ¿Qué elementos podemos analizar en dos fotografías, una rural y otra urbana, que sean singulares del paisaje característico de cada ámbito?

*Concurso de fotografía.
*Visionado de fragmento del documental "Destinos de película...New York" para reflexionar sobre el concepto de Paisaje.

*Trabajo individual: realización de una fotografía de un espacio urbano y analizar los elementos que aparecen en la misma.

*Trabajo en grupo y presentaciones orales de análisis de paisajes urbanos: factores y elementos.

*Autoevaluación individual y en grupo: mediante Kahoot se autoevalúan ellos mismos y también de forma grupal

*Cuestionario final contenidos del bloque, para evaluar la escalera de aprendizaje. 


\section{Semana 18.}

Bloque 6: ¿Qué tipo de problemas (sociales, funcionales, ambientales,) podemos encontrar en los espacios rurales y urbanos?
*Cuestionario previo sobre contenidos del bloque.

*Realización de "lluvia de ideas" sobre los problemas que pueden presentar los ámbitos rurales y urbanos.

*Trabajo individual: búsqueda de noticias periodísticas sobre el contenido del bloque, en un periodo temporal de los últimos 10 años.

*Trabajo en grupo y presentaciones orales problemas agrupados por tipologías en los distintos ámbitos.

\section{Semana 19.}

Bloque 6: ¿Qué tipo de problemas (sociales, funcionales, ambientales,) podemos encontrar en los espacios rurales y urbanos?
* Taller conceptual: realización individual de esquemas de contenidos (conceptuales y procedimentales). Puesta en común, trabajo en grupos y debate.

* Elaboración de murales a partir de los resultados del taller conceptual realizado en la sesión anterior.

*Autoevaluación individual y en grupo: mediante Kahoot se autoevalúan ellos mismos y también de forma grupal

*Cuestionario final contenidos del bloque, para evaluar la escalera de aprendizaje.

*Cuestionario previo sobre contenidos del bloque. *Visionado de documental: "Ordenamiento territorial: qué es y para qué sirve." Debate y reflexión. *Trabajo individual con diferentes documentos de planificación territorial y urbana.

*Trabajo en grupo y presentaciones orales sobre el tratamiento que hacen las distintas Administraciones del sistema de planificación y gestión: análisis de competencias territoriales. 


\section{Semana 21.}

Bloque 7: Ante esos problemas, ¿hay algún sistema de planificación y/o gestión que los trate? ¿Cuáles y en qué forma?
Semana 22.

Salida de campo: Analizamos territorios concretos
* Taller conceptual: realización individual de esquemas de contenidos (conceptuales y procedimentales). Puesta en común, trabajo en grupos y debate.

* Actividad: "Trabajamos en una Consultora". Se trata de realizar el análisis de "caso tipo" y de los procedimientos a seguir con respecto a la gestión territorial.

* Charla-debate con una profesora visitante de Eslovenia, que abre una ventana a la realidad existente en su país.

*Autoevaluación individual y en grupo: mediante Kahoot se autoevalúan ellos mismos y también de forma grupal

*Cuestionario final contenidos del bloque, para evaluar la escalera de aprendizaje.

*Presentación de la guía de trabajo que se utilizará en la salida de campo.

*Trabajo individual previo a la salida: el conocimiento de los espacios. Se eligen los municipios de Brenes (Vega de Sevilla) y Dos Hermanas (Área metropolitana de Sevilla). Búsqueda de información sobre los mismos desde los puntos de vista de situación, demografía, actividad económica, crecimiento urbano y planes de ordenación (territorial y urbanos).

*Trabajo en grupo: puesta en común de información y caracterización de los ámbitos y búsqueda de cartografía sobre los ámbitos.

En esa semana pero fuera del horario de clase: Realización de la salida de campo. Durante la misma deben realizar fotografías y tomar notas de las explicaciones para entregar la semana siguiente una Memoria.

*Repaso general de los contenidos (conceptua-

Semana 23.

Repaso de los contenidos. Cierre de la asignatura les, procedimentales y actitudinales) de toda la asignatura.

*Autoevaluación individual y en grupo: mediante Kahoot se autoevalúan ellos mismos y también de forma grupal

*Cuestionario final contenidos de la asignatura, para evaluar la escalera de aprendizaje. 


\section{Cuestionario inicial y final para hacer un seguimiento de la evolución.}

Como se ha podido observar en el cronograma de actividades, todos los bloques han empezado y terminado con un cuestionario de autoevaluación. El inicial era elaborado por mí, con el objetivo de analizar el punto de partida de cada alumnado y el grado de conocimiento sobre los contenidos a impartir. El cuestionario final era diseñado por "un órgano de trabajo", es decir, un grupo de 6 miembros. Ellos elaboraban el Kahoot que el resto de sus compañeros iban a ir contestando. En cada bloque el encargado de su diseño era un órgano distinto, los cuales elegían un portavoz que era el responsable de desarrollarlo en el aula.
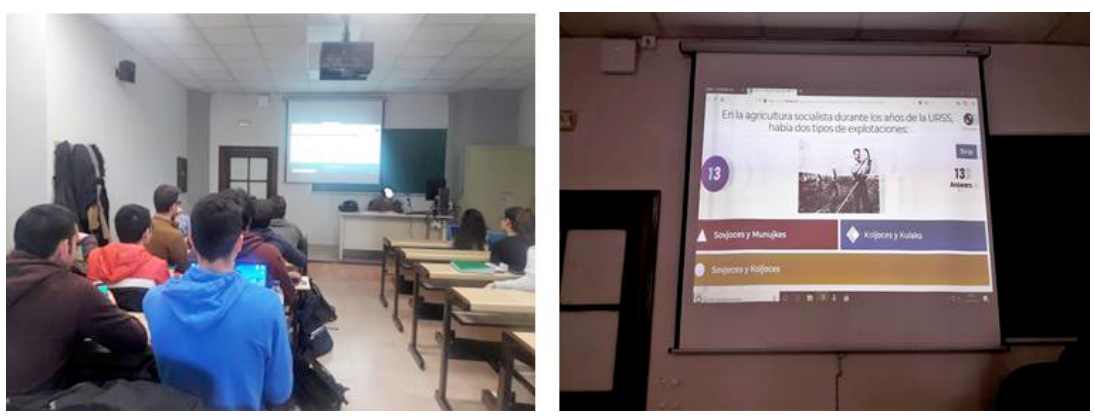

Imagen 1. Realizando actividad de autoevaluación mediante el Kahoot.

\section{Aplicación del Ciclo de Mejora.}

El ciclo de mejora docente (CMD) se ha desarrollado tal y cómo se había diseñado previamente a la puesta en marcha del mismo, siguiendo las pautas, recomendaciones y sugerencias que se aportaron en el curso anterior por parte de los dinamizadores del mismo y de experiencias de propios compañeros.

En un primer momento, se denota cierto nerviosismo por parte del alumnado ante la explicación de las innovaciones docentes metodológicas que se iban a introducir en el desarrollo de la asignatura. Les llamó mucho la atención 
el hecho de que antes de empezar un contenido, se les pasase un cuestionario sobre el conocimiento tanto conceptual como procedimental de los mismos. Sin embargo, el funcionamiento ha sido rápidamente asumido dentro de la normalidad de las clases, con lo que en las semanas finales la dinámica utilizada para trabajar en el aula era ya algo totalmente logrado.

El comenzar normalmente un bloque de trabajo con el visionado de un documental o fragmento de película, ha conseguido despertar, en los momentos iniciales de tener que enfrentarse a una temática desconocida aún por ellos, la curiosidad en los mismos y, más aún, paliar algún leve rechazo a la hora de trabajar temáticas no muy atractivas.

Trabajar agrupados en "órganos "es una de las innovaciones que más han funcionado. No sólo por el reparto de tareas entre ellos mismos y la posibilidad de complementar tiempos de trabajo, sino porque ha permitido fomentar y desarrollar las relaciones interpersonales y consolidarse como grupo (me refiero a la clase entera). En esta cuestión, se han desarrollado de forma importante los contenidos actitudinales, y ha creado un clima de trabajo en el aula realmente positivo para alcanzar los objetivos de la asignatura.

Los talleres conceptuales han servido para afianzar y darle seguridad al alumnado de los contenidos que se estaban dando. Han servido de hilo conductor para la elaboración por parte de los alumnos de sus propios documentos de estudio y, la puesta en común de todos ha complementado la del alumnado cuya evolución en la adquisición de conocimiento se realizaba a un ritmo más lento.

Finalmente, y aunque ya se ha comentado, la posibilidad de que cada alumno/a sea consciente de sus debilidades y fortalezas mediante su propia autoevaluación (por el Kahoot) favorece que pueda ir ajustando su propio proceso de aprendizaje a dichas cuestiones, y por tanto, dedicándole más tiempo a lo deficiente y menos tiempo al contenido ya logrado. 


\section{Evaluación de la puesta en práctica del ciclo de Mejora}

La evaluación del ciclo de mejora permite comprobar cómo ha sido el proceso de aprendizaje del alumnado con respecto al proceso de enseñanza innovador llevado a cabo, saber la opinión del alumnado ante la puesta en marcha de estas acciones distintas a las que han venido desarrollándose tradicionalmente centradas en la exposición magistral de contenidos, y por último, afianzar cuales son aspectos a mantener para los próximos cursos, cuales a modificar o cambiar y afianzar los principios didácticos que son necesario para conseguir que el proceso de enseñanza-aprendizaje funcione de forma más satisfactoria para todos los agentes implicados.

\section{Evaluación del aprendizaje de los estudiantes}

Se pasaron a los alumnos cuestionarios al inicio y al final de la asignatura para poder evaluar su ascenso en la escalera de aprendizaje de los mismos. Para ello se les solicitó que lo firmaran con un pseudónimo. Sólo 32 cuestionarios fueron clasificados como válidos. Se descartaron los de aquellos alumnos a los que le faltaba alguno de los dos (el inicial o final) ya que imposibilitaba la comparación. Las respuestas se evaluaron bajo 4 niveles: respuestas incorrectas o con grandes errores (nivel 1); respuestas incompletas pero muestra cierto conocimiento del contenido (nivel 2); respuestas correctas pero sin concretar demasiado (nivel 3); y finalmente, respuestas completas, bien argumentadas y desarrolladas (nivel 4). Los resultados obtenidos aparecen registrados en la tabla 4.

\section{Tabla 4}

Evaluación de las respuestas de los cuestionarios inicial y final según nivel conseguido por el alumnado. 


\begin{tabular}{|c|c|c|}
\hline $\begin{array}{c}\text { NIVELES ALCANZADOS DE } \\
\text { CONOCIMIENTO }\end{array}$ & $\begin{array}{c}\text { CUESTIONARIO INICIAL } \\
\text { (19 DE MARZO DE 2018) }\end{array}$ & $\begin{array}{c}\text { CUESTIONARIO FINAL } \\
\text { (4 DE JUNIO DE 2018) }\end{array}$ \\
\hline NIVEL 1 & $72,3 \%$ & $16,0 \%$ \\
\hline NIVEL 2 & $25,4 \%$ & $21,9 \%$ \\
\hline NIVEL 3 & $2,3 \%$ & $46,2 \%$ \\
\hline NIVEL 4 & $0 \%$ & $15,9 \%$ \\
\hline
\end{tabular}

Los resultados muestran una favorable evolución en el proceso de enseñanza-aprendizaje del alumnado de la asignatura. Si en el cuestionario inicial el 72,3\% de los mismos se situaban en el nivel 1, por tanto sin ningún o bajo conocimiento sobre los contenidos, en el cuestionario final se reduce al $16 \%$. Lo más llamativo, es que un $46,2 \%$ del alumnado se localiza al final del curso en el nivel 3, y por tanto con las preguntas correctas, aunque sin concretar demasiado. Es decir, que el 66,1\% del alumnado encuestado ha conseguido asumir de forma satisfactoria los contenidos de la asignatura. Esta cuestión se ha reflejado en la evaluación final de la asignatura, donde tan solo un $12,3 \%$ del alumnado no ha superado los contenidos y debe volver a examinarse en Septiembre. Comparando este porcentaje con el del curso 2016/2017 en la misma asignatura (la cual fue impartida por mí), he comprobado que se ha producido un descenso del porcentaje de suspensos, ya que ese curso ascendió a un 29,7\%.

\section{Evaluación del ciclo de mejora por parte de los estudiantes}

Para conocer la evaluación que el alumnado hacía de las distintas acciones innovadoras llevadas a cabo en el CMD, se les pasó un cuestionario con 6 ítems a evaluar, a los que debían dar una puntuación entre 1 y 5 , siendo 1 que le ha parecido una acción mala y 5 una acción excelente. Los resultados pueden observarse en la tabla 5.

Jornadas de Formación e Innovación Docente del Profesorado I № 1 (2018) Esta obra se distribuye con la licencia Creative Commons 
Tabla 5.

Evaluación realizada por el alumnado sobre las acciones innovadoras CMD.

\begin{tabular}{|l|c|}
\hline $\begin{array}{c}\text { ACCIONES REALIZADAS CON METODOLO- } \\
\text { GÍA INNOVADORA }\end{array}$ & $\begin{array}{c}\text { PUNTUACIÓN DEL ALUMNADO } \\
\text { (1-Malo/5-Excelente) }\end{array}$ \\
\hline Cuestionarios iniciales y finales & $\mathbf{4 , 5}$ \\
\hline $\begin{array}{l}\text { Visionado de documentales y fragmen- } \\
\text { tos de películas }\end{array}$ & $\mathbf{3 , 8}$ \\
\hline Trabajar los contenidos en grupo & $\mathbf{4}$ \\
\hline Trabajar fuera del espacio del aula & $\mathbf{4 , 2}$ \\
\hline Realización de los talleres conceptuales & $\mathbf{3 , 4}$ \\
\hline $\begin{array}{l}\text { Salida de Campo: proceso de prepara- } \\
\text { Ción y visitas }\end{array}$ & $\mathbf{3 , 5}$ \\
\hline $\begin{array}{l}\text { EVALUACIÓN GLOBAL DEL CICLO DE MEJORA } \\
\text { DOCENTE }\end{array}$ & $\mathbf{4 , 1}$ \\
\hline
\end{tabular}

De forma global, la evaluación del CMD da un resultado de 4,1, es decir, que el alumnado considera que la aplicación de dichas actividades son muy buenas para el proceso de aprendizaje de ellos. Destacar la gran aceptación de los procesos de autoevaluación y la necesidad de replantear para el próximo curso el método seguido en la realización de los talleres conceptuales.

\section{Conclusiones finales: aspectos a mantener y principios didácticos.}

Como conclusiones finales, hay que destacar que la realización del CDM para la totalidad de la asignatura ha permitido tener una visión más general de lo importante que es introducir distintas acciones innovadoras para mejorar el proceso de enseñanza-aprendizaje, ya que los resultados obtenidos tanto en la evaluación del propio Ciclo por parte del alumnado, como del mismo con respecto a la adquisición de contenidos ha sido muy satisfactorio. 
Para el próximo curso, el objetivo es aplicar el modelo en la totalidad del temario considerado en el diseño. Se van a mantener todos los principios didácticos aplicados ya en éste, aunque hay que revisar de forma más concreta el planteamiento de los talleres conceptuales y la salida de campo. Lo que claramente se va a mantener es el trabajo mediante la fórmula de las denominadas "células de trabajo". El funcionamiento ha favorecido la interrelación alumno-alumno, grupo- grupo y grupo-profesor. Ha sido, por tanto, un motor de motivación durante el trascurso de todas las semanas del CMD y ello ha quedado reflejado en la evaluación final. De forma concreta, para el curso próximo se tendrá en cuenta:

a) En cuanto al aprendizaje de los/as alumnos/as: es fundamental partir del papel de las ideas o modelos mentales de los estudiantes. La conexión con el alumnado desde el principio es una pieza clave para el óptimo desarrollo del proceso.

b) En cuanto a los contenidos de enseñanza: es indispensable realizar una clasificación de los tipos de contenidos, relacionándolos entre ellos, realizando jerarquías según la importancia de los mismos, y seleccionar la forma en que se le va a presentar al alumnado.

c) En cuanto a la metodologia: es necesario diseñar cual será nuestro modelo metodológico personal, las fases que conllevaría la puesta en funcionamiento del mismo, y el modo de plantearlo: preguntas-claves, sub-preguntas, secuencias de actividades, etc.

d) En cuanto a la evaluación: ha quedado comprobado la importancia de un diagnóstico inicial sobre las ideas que a priori el alumnado conoce del tema a tratar, y de las ideas finales o modelos mentales de los estudiantes. Ello permite que de forma rápida podamos ir analizando la evolución que el alumnado va teniendo en 


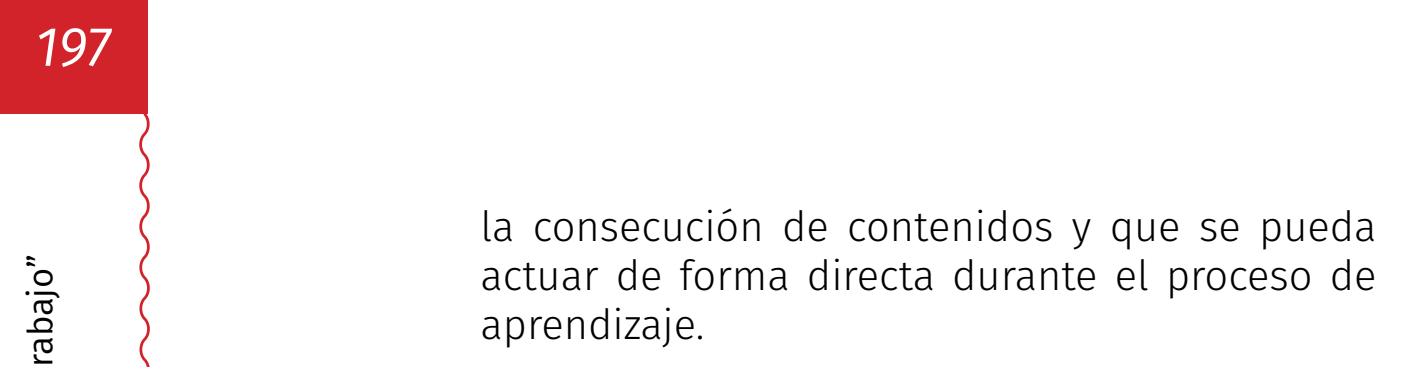

\section{Referencias bibliográficas}

Bain, K. (2007). Lo que hacen los mejores profesores universitarios. Valencia: Publicaciones de la Universidad de Valencia.

Finkel, D. (2008). Dar clase con la boca cerrada. Valencia: Públicaciones de la Universidad de Valencia.

Porlán Ariza, R. (coord.) (2017). Enseñanza Universitaria. Cómo mejorarla. Madrid: Ediciones Morata.

Jornadas de Formación e Innovación Docente del Profesorado | № 1 (2018)

(C) Esta obra se distribuye con la licencia Creative Commons Reconocimiento-NoComercial-SinObraDerivada $\quad 4.0$ 